Kragujevac Journal of Mathematics

Volume 40(1) (2016), Pages 47-59.

\title{
ON PARALLEL RULED SURFACES IN GALILEAN SPACE
}

\author{
M. DEDE ${ }^{1}$ AND C. EKICI ${ }^{2}$
}

\begin{abstract}
In this paper, we investigate the parallel surfaces of the ruled surfaces in Galilean space. There are three types of ruled surfaces in Galilean space. We derive the necessary conditions for each type of the ruled surfaces of the parallel surfaces to be ruled. Consequently, we construct some examples.
\end{abstract}

\section{INTRODUCTION}

A surface formed by a one-parameter family of straight lines is a ruled surface. The geometry of ruled surfaces is essential for many different areas of computer aided design (CAD). Hence, geometers have investigated many properties of the ruled surfaces in both Euclidean and Minkowski spaces [1,5,6,10,14]. In Euclidean case, the parallel surfaces of a ruled surface are in general not ruled surface, but the parallel surface of cylindrical ruled surface are ruled [16]. Recently, Ünlütürk [11] studied parallel ruled surfaces in Minkowski space. In [3], the authors discussed the parallel surfaces in Galilean space.

What is the necessary condition for parallel surface of a ruled surface to be ruled in Galilean space? The purpose of this paper is to give answers to the question mentioned above. The paper is organized as follows: In Section 2 we present the mathematical background on the differential geometry of surfaces in Galilean space. In Sections 3-5 we introduce parallel surfaces to given ruled surfaces of type $A, B$ and $C$. We conclude our paper with two examples.

The Galilean space $\mathbb{G}_{3}$ is a Cayley-Klein space equipped with the projective metric of signature $(0,0,+,+)$. The absolute figure of the Galilean geometry consists of an ordered triple $\{\omega, f, I\}$, where $\omega$ is the real (absolute) plane, $f$ is the real line (absolute line) in $\omega$ and $I$ is the fixed elliptic involution of points of $f$. We introduce

Key words and phrases. Parallel surfaces, Galilean space, ruled surfaces.

2010 Mathematics Subject Classification. Primary: 53A35. Secondary: 53Z05.

Received: July 5, 2015.

Accepted: February 3, 2016. 
homogeneous coordinates in $\mathbb{G}_{3}$ in such a way that the absolute plane $\omega$ is given by $x_{0}=0$, the absolute line $f$ by $x_{0}=x_{1}=0$ and the elliptic involution by

$$
\left(0: 0: x_{2}: x_{3}\right) \rightarrow\left(0: 0: x_{3}:-x_{2}\right) \text {. }
$$

More details about the Galilean and pseudo-Galilean spaces can be found in $[2,4$, $12,13,17,18]$.

Let $\mathbf{a}=(x, y, z)$ and $\mathbf{b}=\left(x_{1}, y_{1}, z_{1}\right)$ be vectors in Galilean space. The scalar product $\mathbf{a}$ and $\mathbf{b}$ is defined by

$$
<\mathbf{a}, \mathbf{b}>=x_{1} x .
$$

The scalar product of two isotropic vectors, $\mathbf{p}=(0, y, z)$ and $\mathbf{q}=\left(0, y_{1}, z_{1}\right)$, is given by

$$
<\mathbf{p}, \mathbf{q}>_{1}=y y_{1}+z z_{1} .
$$

The cross product of the vectors $\mathbf{u}=\left(u_{1}, u_{2}, u_{3}\right)$ and $\mathbf{v}=\left(v_{1}, v_{2}, v_{3}\right)$ is defined as follows:

$$
\mathbf{u} \wedge \mathbf{v}=\left|\begin{array}{ccc}
0 & e_{2} & e_{3} \\
u_{1} & u_{2} & u_{3} \\
v_{1} & v_{2} & v_{3}
\end{array}\right|=\left(0, u_{3} v_{1}-u_{1} v_{3}, u_{1} v_{2}-u_{2} v_{1}\right)
$$

where $e_{2}$ and $e_{3}$ are Euclidean standard basis.

\section{Surface Theory in Galilean Space}

Let $M$ be a surface given by the parametrization

$$
\varphi\left(v^{1}, v^{2}\right)=\left(x\left(v^{1}, v^{2}\right), y\left(v^{1}, v^{2}\right), z\left(v^{1}, v^{2}\right)\right), \quad v^{1}, v^{2} \in \mathbb{R},
$$

where $x\left(v^{1}, v^{2}\right), y\left(v^{1}, v^{2}\right), z\left(v^{1}, v^{2}\right) \in C^{3}[15]$. We denote the partial derivatives of $\varphi\left(v^{1}, v^{2}\right)$ with respect to $v^{1}$ and $v^{2}$ by $\varphi_{, 1}\left(v^{1}, v^{2}\right)$ and $\varphi_{, 2}\left(v^{1}, v^{2}\right)$.

The isotropic unit normal vector field $\mathbf{N}$ is given by

$$
\mathbf{N}=\frac{\varphi, 1 \wedge \varphi_{, 2}}{w}
$$

where $w=\left\|\varphi, 1 \wedge \varphi_{, 2}\right\|_{1}$.

The first fundamental form $I$ of the surface is given by

$$
I=\left(g_{i j}+\epsilon h_{i j}\right) d v^{i} d v^{j}
$$

where $h_{i j}$ and $g_{i j}(i, j=1,2)$ are called the induced metric on the surface given by

$$
h_{i j}=\left\langle\varphi_{, i}, \varphi_{, j}\right\rangle_{1}, \quad g_{i j}=\left\langle\varphi_{, i}, \varphi_{, j}\right\rangle
$$

and

$$
\epsilon=\left\{\begin{array}{lll}
0, & d v^{1}: d v^{2} & \text { non-isotropic } \\
1, & d v^{1}: d v^{2} & \text { isotropic. }
\end{array}\right.
$$

The coefficients $L_{i j}$ of the second fundamental form are given by

$$
L_{i j}=\left\langle\frac{\varphi_{, i j} x_{, 1}-x_{, i j} \varphi_{, 1}}{x_{, 1}}, \mathbf{N}\right\rangle_{1} .
$$


The Gaussian curvature $K$ and the mean curvature $H$ of the surface are given by

$$
K=\frac{\operatorname{det} L_{i j}}{w^{2}}, \quad 2 H=g^{i j} L_{i j},
$$

where

$$
g^{1}=\frac{x_{, 2}}{w}, \quad g^{2}=-\frac{x_{, 1}}{w}, \quad g^{i j}=g^{i} g^{j} .
$$

Note that $x_{, 1}$ and $x_{, 2}$ are the partial derivatives of the first component of the surface given in (2.1).

Definition 2.1. Let $M$ be a surface in Galilean space. We define the parallel surface $M^{\lambda}$ to base surface $M$ at constant distance scalar $\lambda$ as

$$
\varphi^{\lambda}(x, u)=\varphi(x, u)+\lambda \mathbf{N}(x, u),
$$

where $\mathbf{N}$ is the normal vector of the base surface.

Theorem 2.1. Let $M$ and $M^{\lambda}$ be two parallel surfaces in $\mathbb{G}_{3}$. The relations between the Gaussian curvatures and the mean curvatures of two parallel surfaces are

$$
K^{\lambda}=\frac{K}{1-2 \lambda H}
$$

and

$$
H^{\lambda}=\frac{H}{1-2 \lambda H}
$$

respectively.

Definition 2.2. A ruled surface is called developable if and only if the parameter of distribution vanishes.

\section{Parallel Surface of Ruled Surface of Type $A$}

There are three types of ruled surfaces in $\mathbb{G}^{3}$. Here we may distinguish the parallel surfaces of each types of the ruled surfaces. We recall the following definitions, given in [7-9]. A ruled surface of type $A$ in $\mathbb{G}_{3}$ can be parametrized by

$$
\Phi_{A}(x, u)=m(x)+u a(x),
$$

where the curve $m(x)=(x, y(x), z(x))$ is called a directrix does not lie in a Euclidean plane and the generators $a(x)=\left(1, a_{2}(x), a_{3}(x)\right)$ are non-isotropic.

The associated orthonormal trihedron is defined by

$$
\begin{aligned}
\mathbf{t}(x) & =\left(1, a_{2}(x), a_{3}(x)\right), \\
\mathbf{n}(x) & =\frac{1}{\kappa}\left(0, a_{2}^{\prime}(x), a_{3}^{\prime}(x)\right), \\
\mathbf{b}(x) & =\frac{1}{\kappa}\left(0,-a_{3}^{\prime}(x), a_{2}^{\prime}(x)\right),
\end{aligned}
$$


where $\kappa=\sqrt{\left(a_{2}^{\prime}\right)^{2}+\left(a_{3}^{\prime}\right)^{2}}$. Frenet formulas are given as follows

$$
\frac{d}{d x}\left[\begin{array}{l}
\mathbf{t} \\
\mathbf{n} \\
\mathbf{b}
\end{array}\right]=\left[\begin{array}{ccc}
0 & \kappa & 0 \\
0 & 0 & \tau \\
0 & -\tau & 0
\end{array}\right]\left[\begin{array}{l}
\mathbf{t} \\
\mathbf{n} \\
\mathbf{b}
\end{array}\right],
$$

where $\tau=\frac{\operatorname{det}\left(a, a^{\prime}, a^{\prime \prime}\right)}{\kappa^{2}}$ is called the torsion.

The parameter of distribution $d$ is given by

$$
d=-\frac{\operatorname{det}\left(m^{\prime}, a, a^{\prime}\right)}{\kappa} .
$$

A simple calculation implies that

$$
m^{\prime}(x)=\mathbf{t}-\kappa d \mathbf{b} .
$$

The unit normal vector is

$$
\mathbf{N}(x, u)=\frac{d \mathbf{n}+u \mathbf{b}}{\sqrt{d^{2}+u^{2}}} .
$$

The Gaussian curvature $K$ and the mean curvature $H$ are given by

$$
K=-\frac{d^{2}}{\left(d^{2}+u^{2}\right)^{2}}
$$

and

$$
2 H=-\frac{d^{\prime} \kappa u-\tau \kappa^{\prime} d^{2}+\kappa d-\kappa \tau u^{2}}{\kappa^{2}\left(d^{2}+u^{2}\right)^{\frac{3}{2}}},
$$

respectively.

We consider now the parallel surface of the ruled surface of type $A$. Substituting (3.1) and (3.4) into (2.8), we obtain the parallel surface $\Phi_{A}^{\lambda}$ to given ruled surface $\Phi_{A}$ in the following form

$$
\Phi_{A}^{\lambda}(x, u)=m+\lambda \frac{d \mathbf{n}}{\sqrt{d^{2}+u^{2}}}+u\left(a+\lambda \frac{\mathbf{b}}{\sqrt{d^{2}+u^{2}}}\right) .
$$

This means that $\Phi_{A}^{\lambda}$ is not generally a ruled surface. Thus, we give the following corollary.

Corollary 3.1. If the ruled surface of type $A$ is developable $(d=0)$, then the parallel surface of the ruled surface is ruled.

It follows that, the parallel ruled surface is parametrized by

$$
\Phi_{A}^{\lambda}(x, u)=m^{\lambda}(x)+u a(x),
$$

where $m^{\lambda}(x)$ the directrix of the parallel surface is given by

$$
m^{\lambda}(x)=m(x)+\lambda \mathbf{b} .
$$


We also want to point out that since $m^{\lambda}(x)$ the directrix of parallel ruled surface does not lie in a Euclidean plane and the generator of both ruled surfaces is also identical, the parallel ruled surface is also ruled surface of type $A$.

From (3.2) and (3.3), the derivative $m^{\lambda \prime}$ may be calculated as

$$
m^{\lambda \prime}=\mathbf{t}-\lambda \tau \mathbf{n} \text {. }
$$

It is easy to see that the parameter of distribution of the parallel ruled surface is

$$
d^{\lambda}=0 \text {. }
$$

Hence, we state the following corollary.

Corollary 3.2. If the ruled surface of type $A$ is developable, then the parallel ruled surface is also developable.

On the other hand, the partial derivatives of the curvatures of $\Phi_{A}^{\lambda}$ with respect to $x$ and $u$ are obtained in the following form

$$
\frac{\partial \Phi_{A}^{\lambda}(x, u)}{\partial x}=\mathbf{t}+(u \kappa-\lambda \tau) \mathbf{n}, \quad \frac{\partial \Phi_{A}^{\lambda}(x, u)}{\partial u}=\mathbf{t} .
$$

By using (2.2) and (3.7), we have

$$
w^{\lambda}=u \kappa-\lambda \tau
$$

Hence, the unit normal vector is obtained as follows

$$
\mathbf{N}^{\lambda}=\mathbf{b} \text {. }
$$

Now we turn our attention to the coefficients of the first fundamental form of parallel ruled surface. First of all, it is easy to see that the first component of the parallel ruled surface of type $\mathrm{A}$ is

$$
\Phi_{A}^{\lambda}(x, u)=(x+u, \ldots, \ldots) .
$$

Then, from (1.2), (2.4) and (3.10), we get

$$
\left(g_{11}\right)^{\lambda}=\left(g_{12}\right)^{\lambda}=\left(g_{22}\right)^{\lambda}=1 .
$$

Using the projection of $\frac{\partial \Phi_{A}^{\lambda}(x, u)}{\partial x}$ and $\frac{\partial \Phi_{A}^{\lambda}(x, u)}{\partial x}$ vectors onto the Euclidean $y z$-plane and (2.4), we have

$$
h_{11}=1+(u \kappa-\lambda \tau)^{2}, \quad h_{12}=h_{22}=1 .
$$

Then, substituting (3.11) and (3.12) into (2.3), we obtain the first fundamental form of the parallel ruled surface of type $A$ as

$$
I^{\lambda}=(d x+d u)^{2}+\epsilon(u \kappa-\lambda \tau)^{2} d u^{2},
$$

where

$$
\epsilon=\left\{\begin{array}{lll}
0, & d x \neq-d u & \text { non-isotropic } \\
1, & d x=-d u & \text { isotropic. }
\end{array}\right.
$$


By using (3.9) and the partial derivatives of (3.7), we obtain the coefficients of the second fundamental form as follows

$$
L_{11}^{\lambda}=u \kappa \tau-\lambda \tau^{2}, \quad L_{12}^{\lambda}=L_{22}^{\lambda}=0 .
$$

Hence we state the following theorem.

Theorem 3.1. Let $\Phi_{A}$ and $\Phi_{A}^{\lambda}$ be two parallel ruled surfaces of type $A$ in $\mathbb{G}_{3}$. The Gaussian and the mean curvatures of $\Phi_{A}^{\lambda}$ can be obtained in terms of the curvature and torsion of $\Phi_{A}$ as follows

$$
K^{\lambda}=0
$$

and

$$
H^{\lambda}=\frac{\tau}{2(\kappa u-\lambda \tau)}
$$

Proof. Using (2.6), (3.8) and (3.13) gives

$$
K^{\lambda}=0 .
$$

Substituting (3.10) and (3.8) into (2.7) implies that

$$
\left(g^{11}\right)^{\lambda}=\left(g^{22}\right)^{\lambda}=\frac{1}{(u \kappa-\lambda \tau)^{2}}
$$

and

$$
\left(g^{12}\right)^{\lambda}=-\frac{1}{(u \kappa-\lambda \tau)^{2}}
$$

Combining (2.6), (3.13), (3.17) and (3.18) yields

$$
H^{\lambda}=\frac{\tau}{2(\kappa u-\lambda \tau)} \text {. }
$$

Finally, we remark that the formulas given in (3.14) and (3.15) can be obtained in a different way. Using $d=0,(2.9),(2.10),(3.5)$ and (3.6) gives the same results.

\section{Parallel Surface of Ruled Surface of Type $B$}

A ruled surface of type $B$ can be parametrized by

$$
\Phi_{B}(x, u)=r(x)+u a(x),
$$

where its striction curve $r(x)=(0, y(x), z(x))$ lies in a Euclidean plane and $a(x)=$ $\left(1, a_{2}(x), a_{3}(x)\right)$ is the generator.

The associated trihedron of the ruled surfaces of type $B$ is defined by

$$
\begin{aligned}
& \mathbf{t}(x)=\left(1, a_{2}(x), a_{3}(x)\right), \\
& \mathbf{n}(x)=\left(0,-z^{\prime}(x), y^{\prime}(x)\right), \\
& \mathbf{b}(x)=\left(0, y^{\prime}(x), z^{\prime}(x)\right) .
\end{aligned}
$$


Then the Frenet formulas are

$$
\frac{d}{d x}\left[\begin{array}{l}
\mathbf{t} \\
\mathbf{n} \\
\mathbf{b}
\end{array}\right]=\left[\begin{array}{ccc}
0 & \kappa & 0 \\
0 & 0 & \tau \\
0 & -\tau & 0
\end{array}\right]\left[\begin{array}{l}
\mathbf{t} \\
\mathbf{n} \\
\mathbf{b}
\end{array}\right],
$$

where $\kappa$ is the curvature and $\tau$ is the torsion.

It is easy to see that the parameter of distribution is $d=\frac{1}{\kappa}$. The unit normal vector $\mathbf{N}$ is obtained by

$$
\mathbf{N}=\frac{-\mathbf{n}+u \kappa \mathbf{b}}{\sqrt{1+\kappa^{2} u^{2}}}
$$

The Gaussian and mean curvatures are given by

$$
K=-\frac{\kappa^{2}}{\left(1+\kappa^{2} u^{2}\right)^{2}}
$$

and

$$
H=\frac{\tau-u \kappa^{\prime}+u^{2} \kappa^{2} \tau}{2\left(1+\kappa^{2} u^{2}\right)^{\frac{3}{2}}},
$$

respectively.

Substituting (4.1) and (4.4) into (2.8) implies that

$$
\Phi_{B}^{\lambda}(x, u)=r(x)-\lambda \frac{\mathbf{n}}{\sqrt{1+\kappa^{2} u^{2}}}+u\left(a(x)+\lambda \frac{\kappa \mathbf{b}}{\sqrt{1+\kappa^{2} u^{2}}}\right) .
$$

Observe that $\Phi_{B}^{\lambda}$ is not a ruled surface. Hence we have the following corollary.

Corollary 4.1. If the curvature $\kappa$ of the ruled surface of type $B$ is zero, then the parallel surface of ruled surface is also ruled. Moreover $\kappa=0$ means that the generator $a(x)$ of parallel ruled surface of type $B$ is a constant unit vector.

On the other hand, the parallel ruled surface of type $B$ can be parametrized by

$$
\Phi_{B}^{\lambda}(x, u)=r^{\lambda}(x)+u a(x),
$$

where $a(x)$ is the generator and directrix is

$$
r^{\lambda}(x)=r(x)-\lambda \mathbf{n} .
$$

From (4.2), (4.3) and (4.7), we have the derivative of directrix in the following form

$$
r^{\lambda \prime}=(1-\lambda \tau) \mathbf{b} .
$$

The partial derivatives of $\Phi_{B}^{\lambda}$ with respect to $x$ and $u$ are

$$
\frac{\partial \Phi_{B}^{\lambda}(x, u)}{\partial x}=(1-\lambda \tau) \mathbf{b}, \quad \frac{\partial \Phi_{B}^{\lambda}(x, u)}{\partial u}=\mathbf{t} .
$$

By using (2.2), we have

$$
w^{\lambda}=1-\lambda \tau \text {. }
$$


Thus, the unit normal vector is

$$
\mathbf{N}^{\lambda}=\mathbf{n} .
$$

The first component of the parallel ruled surface of type $B$ is

$$
\Phi_{A}^{\lambda}(x, u)=(u, \ldots, \ldots) .
$$

Then, by using (2.4), (2.7), (4.9) and (4.12) we get

$$
\left(g_{11}\right)^{\lambda}=\left(g_{12}\right)^{\lambda}=0,\left(g_{22}\right)^{\lambda}=1
$$

and

$$
h_{11}^{\lambda}=(1-\lambda \tau)^{2} .
$$

Substituting (4.13) and (4.14) into (2.3) gives

$$
I^{\lambda}=d u^{2}+\epsilon(1-\lambda \tau)^{2} d x^{2},
$$

where

$$
\epsilon=\left\{\begin{array}{lll}
0, & d u \neq 0 & \text { non-isotropic } \\
1, & d u=0 & \text { isotropic }
\end{array}\right.
$$

By using the derivatives of (4.9), (4.11) and (2.5), the coefficients of the second fundamental form are obtained as

$$
L_{11}^{\lambda}=(1-\lambda \tau) \tau, \quad L_{12}^{\lambda}=L_{22}^{\lambda}=0 .
$$

Theorem 4.1. Let $\Phi_{B}$ and $\Phi_{B}^{\lambda}$ be two parallel ruled surfaces in $\mathbb{G}_{3}$. The Gaussian and the mean curvatures of $\Phi_{B}^{\lambda}$ may be calculated in terms of the curvature and torsion of $\Phi_{B}$ as

$$
K^{\lambda}=0
$$

and

$$
2 H^{\lambda}=\frac{\tau}{1-\lambda \tau} .
$$

Proof. Using (2.6), (4.10) and (4.15) gives

$$
K^{\lambda}=0 .
$$

Substituting (4.10) and (4.12) into (2.7) implies that

$$
\left(g^{11}\right)^{\lambda}=\frac{1}{(1-\lambda \tau)^{2}}
$$

and

$$
\left(g^{12}\right)^{\lambda}=\left(g^{22}\right)^{\lambda}=0 .
$$

From (2.6), (4.15), (4.17) and (4.16) we have

$$
2 H^{\lambda}=\frac{\tau}{1-\lambda \tau} .
$$

On the other hand, using $\kappa=0,(2.9)$ and (4.5) gives the same results. 


\section{Parallel Surface of Ruled Surface of Type $C$}

A ruled surface of type $C$ can be parametrized by

$$
\Phi_{C}(x, u)=r(x)+u a(x),
$$

where $r(x)=(x, y(x), 0)$ is called the directrix and $a(x)=\left(0, a_{2}(x), a_{3}(x)\right)$ is the generator.

The associated orthonormal trihedron is given by

$$
\begin{aligned}
\mathbf{t} & =\left(1, y^{\prime}(x), 0\right), \\
\mathbf{n} & =\left(0, a_{2}(x), a_{3}(x)\right), \\
\mathbf{b} & =\left(0,-a_{3}(x), a_{2}(x)\right) .
\end{aligned}
$$

Let $\theta$ be the Euclidean angle between $z=0$ plane and $\mathbf{n}$. Then, Frenet formulas are

$$
\frac{d}{d x}\left[\begin{array}{l}
\mathbf{t} \\
\mathbf{n} \\
\mathbf{b}
\end{array}\right]=\left[\begin{array}{ccc}
0 & \kappa \cos \theta & -\kappa \sin \theta \\
0 & 0 & \frac{1}{\delta} \\
0 & -\frac{1}{\delta} & 0
\end{array}\right]\left[\begin{array}{l}
\mathbf{t} \\
\mathbf{n} \\
\mathbf{b}
\end{array}\right],
$$

where $\kappa=y^{\prime \prime}$ is the curvature and $\delta=-\frac{a_{3}}{a_{2}^{\prime}}$ is the parameter of distribution.

A simple calculation implies that

$$
r^{\prime}(x)=\mathbf{t}, \quad \mathbf{N}=\mathbf{b} .
$$

The Gaussian and mean curvatures are given by

$$
K=-\frac{1}{\delta^{2}}, \quad 2 H=0 .
$$

Substituting (5.1) and (5.3) into (2.8), we get the parallel surface of the ruled surface of type $C$ in the following form

$$
\Phi_{C}^{\lambda}(x, u)=r(x)+\lambda \mathbf{b}+u a(x) .
$$

Observe that the generator $a(x)$ is identical and the directrix is given by

$$
r^{\lambda}(x)=r(x)+\lambda \mathbf{b}
$$

Hence, the following corollary can be given.

Corollary 5.1. The parallel surface of a ruled surface of type $C$ is also a ruled surface.

On the other hand, from (5.2) and (5.5) we have the derivative of the directrix in the following form

$$
r^{\lambda \prime}=\mathbf{t}-\lambda \frac{1}{\delta} \mathbf{n} .
$$


The parameter of distribution of the parallel ruled surface is $d^{\lambda}=d=\delta$. The partial derivatives of $\Phi_{C}^{\lambda}$ with respect to $x$ and $u$ are obtained by

$$
\frac{\partial \Phi_{C}^{\lambda}(x, u)}{\partial x}=\mathbf{t}-\lambda \frac{1}{\delta} \mathbf{n}+u \frac{1}{\delta} \mathbf{b}, \quad \frac{\partial \Phi_{C}^{\lambda}(x, u)}{\partial u}=\mathbf{n} .
$$

Thus,

$$
w^{\lambda}=1
$$

The unit normal vector $\mathbf{N}^{\lambda}$ is

$$
\mathbf{N}^{\lambda}=\mathbf{b}
$$

By using (2.4), (2.7) and (5.6) we have

$$
\left(g_{11}\right)^{\lambda}=1, \quad\left(g_{12}\right)^{\lambda}=\left(g_{22}\right)^{\lambda}=0
$$

and

$$
h_{22}^{\lambda}=1 \text {. }
$$

Substituting (5.9) and (5.10) into (2.3) gives

$$
I^{\lambda}=d x^{2}+\epsilon d u^{2}
$$

where

$$
\epsilon=\left\{\begin{array}{lll}
0, & d x \neq 0 & \text { non-isotropic } \\
1, & d x=0 & \text { isotropic }
\end{array}\right.
$$

Using (5.8), the derivatives of (5.6) and (2.5), the coefficients of the second fundamental form of the parallel ruled surface of type $C$ are obtained by

$$
L_{11}^{\lambda}=\kappa \sin \theta-\lambda \frac{1}{\delta^{2}}+u \frac{1}{\delta^{\prime}}, \quad L_{12}^{\lambda}=\frac{1}{\delta}, \quad L_{22}^{\lambda}=0 .
$$

Theorem 5.1. Let $\Phi_{C}$ and $\Phi_{C}^{\lambda}$ be two parallel ruled surfaces in $\mathbb{G}_{3}$. The Gaussian and the mean curvatures of $\Phi_{C}^{\lambda}$ are calculated as

$$
K^{\lambda}=-\frac{1}{\delta^{2}}, \quad 2 H^{\lambda}=0 .
$$

Proof. Substituting (5.7) and (5.12) into (2.6) gives

$$
K^{\lambda}=-\frac{1}{\delta^{2}} .
$$

By using (5.7) and (2.7) implies that

$$
\left(g^{11}\right)^{\lambda}=\left(g^{12}\right)^{\lambda}=0,\left(g^{22}\right)^{\lambda}=1 .
$$

From (2.6), (5.15) and (5.12) we have

$$
2 H^{\lambda}=0 .
$$

Note that substituting (5.4) into (2.9) and (2.10), gives the same results.

Finally, we can give the following corollary. 


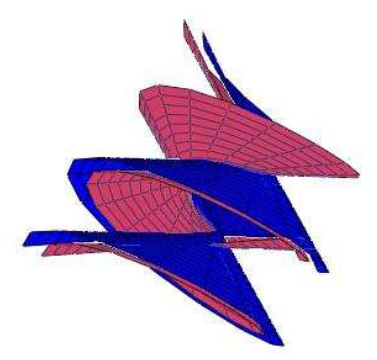

FigURE 1.

Corollary 5.2. The parallel ruled surface of each type is a Weingarten surface in Galilean space.

\section{EXAMPLES}

Example 6.1. Consider the developable ruled surface of type $A$ parametrized by

$$
\Phi_{A}(x, u)=(x+u, \cos x-u \sin x, \sin x+u \cos x) .
$$

The associated trihedron is obtained by

$$
\begin{aligned}
\mathbf{t} & =(1,-\sin x, \cos x), \\
\mathbf{n} & =(0,-\cos x,-\sin x), \\
\mathbf{b} & =(0, \sin x,-\cos x),
\end{aligned}
$$

where $\kappa=\tau=1$. The unit normal is

$$
\mathbf{N}=(0,-\sin x, \cos x) .
$$

For $\lambda=1$, the parallel ruled surface is parametrized by

$$
\Phi_{A}^{\lambda}(x, u)=(x+u,-\sin x+\cos x-u \sin x, \cos x+\sin x+u \cos x),
$$

where the directrix and the generator are given by

$$
m^{\lambda}(x)=(x,-\sin x+\cos x, \cos x+\sin x)
$$

and

$$
a^{\lambda}(x)=(1,-\sin x, \cos x) .
$$

The Gaussian and mean curvatures of the parallel ruled surface are obtained as

$$
K^{\lambda}=0, \quad H^{\lambda}=-\frac{1}{1+v} .
$$

The ruled surface (red) and the parallel ruled surface (blue) are illustrated in Figure 1. 


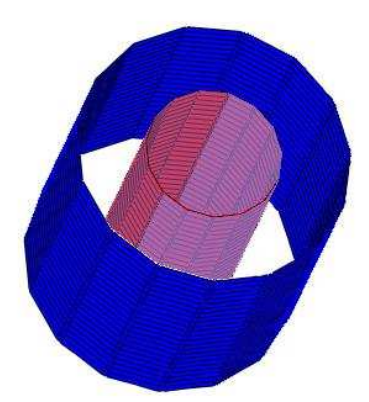

FiguRE 2.

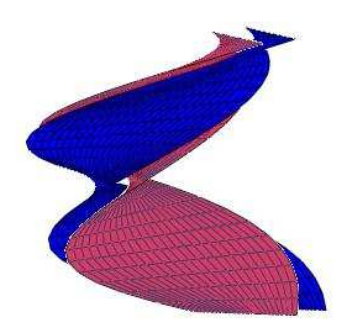

FiguRE 3.

Example 6.2. Assume that the ruled surface of type $B$ is parametrized by

$$
\Phi_{B}(x, u)=(u, \cos x, \sin x),
$$

where $r(x)=(0, \cos x, \sin x)$ is the directrix and $a(x)=(1,0,0)$ is the generator.

It is easy to see that $\kappa=0, \tau=1$. Thus, the parallel ruled surface is obtained by

$$
\Phi_{B}^{\lambda}(x, u)=(u, 2 \cos x, 2 \sin x) .
$$

Figure 2 shows the ruled surface (red) and the parallel ruled surface (blue) in Galilean space.

Example 6.3. Assume that the ruled surface of type $C$ is parametrized by

$$
\Phi_{C}(x, u)=\left(x, x^{2}+u \cos x, u \sin x\right),
$$

where $r(x)=\left(x, x^{2}, 0\right)$ is the directrix and $a(x)=(0, \cos x, \sin x)$ is the generator. For $\lambda=1$, the parallel ruled surface is parametrized by

$$
\Phi_{C}^{\lambda}(x, u)=\left(x,-\sin x+x^{2}+u \cos x, \cos x+u \sin x\right) .
$$

The ruled surface (red) and the parallel ruled surface (blue) are illustrated in Figure 3. 


\section{REFERENCES}

[1] A. C. Çöken, Ü. Çiftçi and C. Ekici, On parallel timelike ruled surfaces with timelike rulings, Kuwait J. Sci. 35 (2008), 21-31.

[2] Z. Milin Sipus and B. Divjak, Translation surfaces in the Galilean space, Glas. Mat. 46 (2011), 455-469.

[3] M. Dede, C. Ekici and A. C. Çöken, On the parallel surfaces in Galilean space, Hacet. J. Math. Stat. 18 (2013), 209-217.

[4] C. Ekici and M. Dede, On the Darboux vector of ruled surfaces in pseudo-Galilean space, Math. Comput. Appl. 16 (2011), 830-838.

[5] H. W. Guggenheimer, Differential Geometry, McGraw-Hill, New York, 1963.

[6] M. K. Sağel and H. H. Hacısalihoğlu, On the parallel hypersurfaces with constant curvature, Commun. Fac. Sci. Univ. Ank. Series A 40 (1991), 1-5.

[7] B. Divjak and Z. Milin-Sipus, Transversal surfaces of ruled surfaces in pseudo Galilean space, Sitzungsber. Abt. II, 213 (2004), 23-32.

[8] Z. Milin-Sipus, Ruled Weingarten surfaces in Galilean space, Period. Math. Hungar. 56 (2008), 213-225.

[9] Z. Milin-Sipus and B. Divjak, Special curves on ruled surfaces in Galilean and pseudo-Galilean spaces, Acta Math. Hungar. 98 (2003), 203-215.

[10] C. Ekici and A. C. Çöken, The integral invariants of parallel timelike ruled surfaces, J. Math. Anal. Appl. 393 (2012), 97-107.

[11] Y. Ünlütürk and C. Ekici, Parallel surfaces of spacelike ruled Weingarten surfaces in Minkowski 3-space, New Trends in Math. Sci. 1 (2013), 85-92.

[12] O. M. Röschel, Die Geometrie des Galileischen Raumes, Habilitationsschrift, Leoben, 1984.

[13] I. M. Yaglom, A Simple Non-Euclidean Geometry and its Physical Basis, Springer-Verlag, New York, 1979.

[14] A. Görgülü and A. Çöken, The Euler theorem for parallel pseudo-Euclidean hypersurfaces in pseudo-Euclidean space, J. Inst. Math. Comput. Sci. Ser. 6 (1993), 161-165.

[15] M. Dede, Tubular surfaces in Galilean space, Math. Commun. 18 (2013), 209-217.

[16] K. R. Park and G. I. Kim, Offsets of ruled surfaces, J. Korean Computer Graphics Society 4 (1998), 69-75.

[17] M. Dede, On the parallel ruled Weingarten surfaces in 3-Dimensional Galilean Space, PhD thesis, Eskisehir Osmangazi University, Eskisehir (in Turkish), 2011.

[18] A. O. Ögrenmis, H. Öztekin and M. Ergut, Bertrand curves in Galilean space and their characterizations, Kragujevac J. Math. 32 (2009), 139-147.

[19] U. Z. Savci , A. Görgülü and C. Ekici, Parallel surfaces of ruled Weingarten surfaces, NTMSCI, 3 (2015), 237-246.

${ }^{1}$ Department of Mathematics,

Kilis 7 ARALIK UNiversity,

E-mail address: mustafadede@kilis.edu.tr

${ }^{2}$ Department of Mathematics-Computer,

ESKIŞEHIR OSMANGAZI UNIVERSITY,

E-mail address: cekici@ogu.edu.tr 